\title{
A reality and the future of the consumer education to the elderly and with slight dementia
}

\author{
Kenji Ushizawa $^{1} \cdot$ Shigeo Takizawa $^{2}$ \\ ${ }^{1}$ School of Management, SANNO University \\ ${ }^{2}$ Biophilia Institute
}

\begin{abstract}
We have researched the reality and the future of the consumer education to the elderly as well as elderly with slight dementia supported by the Grant of Japanese Ministry of Education, Culture, Sports, Science and Technology [MET]. We executed the questionnaire investigation and the evaluation for the experiments. We report in this paper about those contents, the results of the mail survey and the future way of advancing our plan. The mail survey shows that (a) social welfare facilities will be short, therefore a consumption education for the elderly will be needed, and also (b) we should enrich the nursing care of the society and increase the number of people talented for welfare and nursing care.
\end{abstract}

Key words: Consumer education, elderly, slight dementia, questionnaire survey, Takizawa program

\section{Introduction}

As many of us may know, aging of the society is occurring at a rapid pace in Japan. At the same time, the number of elderly in need of nursing care is steadily increasing, and it is presumed to rise to 5.2 million in 2025, and of it 2.3 million bedridden. In addition with the declining birthrates far beyond prospective, Japanese public finance is reaching the crisis of failure. This problem is now a haste and most important political subject in Japan.

With such background, we started a research with the grant of the MET.

The purpose of our research is not a calculation of the elderly in need of nursing care, nor its countermeasure. But our research is on how to not make the elderly in need of nursing care in the first place, moreover, to propose a scheme or methods for the elderly to participate in society as consumers, and to do a fundamental research for those. With it becoming possible, we will build a sustainable life independent society, and are thinking to be able to assist the reactivation of economy.

Our research is composed of three substitute terms. The first is a questionnaire about the present consumption education to the elderly and its perspective to the social welfare facilities of over Japan. We will report the contents afterwards. The second is the improvement and the trial of new evaluation chart referring to the rehabilitation effect of daily life activity, and that it has actually been tried out. And as for the third, we did the experiment of consumption education rehabilitation to the elderly who are being cared for at the day nursing and care facility. In this paper, we report the first substitute term.

\section{Questionnaire survey of the elderly consumer education}

Mail survey was carried out for a month from March to April in 2003 to the social welfare facilities. It was mailed to about 2000 places. Also, the Internet survey with the same content was done for three months from July 
to September. The letters for request were sent to about 2000 facilities.

About the mail survey, almost 250 effective affirmative responses were collected. A collection rate is about $13 \%$. This rate is not so sufficient statistically. However, we believe that respondents of our survey are concerned highly and therefore their opinions will give us effective suggestions to our subjects, that is, the future of the consumer education to the elderly and with slight dementia.

And as for the Internet survey, although about 2000 cooperation requests were sent after the establishment of the homepage, only 20 were collected. This could be said as a present condition of Japan according to the Internet survey. It is not certain whether or not Internet is used in 2000 facilities where the requests were mailed. Also, as the Internet survey, the number of questions might have been too many. This is one of our future examination subjects.

\section{Results of mail survey}

In this chapter we express the main results based the mail survey.

\subsection{The perspective of nursing care and welfare}

Figure 1 shows the survey result of the perspective of nursing care and welfare as the future social conditions.

First, we will pay attention to the consumption problem and the consumer education, which are our research subjects.

Regarding "5. The cases of elderly involved in a consumption trouble will increase more and more", about $80 \%$ replied to agree. It also shows high rate of around $75 \%$ according to "4. The social security-related expenditure will crunch, and enough nursing care will be unavailable in the facilities". Of course, these results show that the independence of elderly is needed. Also, we can rate this as the relation of $70 \%$ to "10. Education is needed for elderly to live independently as one consumer".

What else could be paid attention is nearly $90 \%$ affirmative opinion on "7. More people to carry nursing care and welfares are needed", or "8. Society nursing care facilities with autonomous communities (city and else) as the leader should be productive", which marked high rate of $85 \%$.

\subsection{Present condition of consumer education}

Then, as a present condition, what are the social welfare facilities in track of the elderly consumption problems or consumption activities? The figure 2 will show its present condition.

As the term 8 shows, we can see that the $50 \%$ of the facilities are not mentioning about the consumption activities or shopping activities. Also, considering this subject, although 40 to $50 \%$ of them are listening and communicating with users or his/her family, the number of the facilities that are organizing the fact as a data is very low. Therefore, we may understand that at present condition, the facts gained from elderly consumption activities or shopping activities, are not organized enough as a data. 
1. It is possible to admit in the facilities about most of the disabled elderly who will want to do it from now on.

2. Present measure to certification of long-term care need should be reconsidered.

3. The policies, which it gives priority to care for home over, must be taken.

4.The social security-related expenditure will crunch, and enough nursing care will be unavailable in the facilities.

5.The cases of elderly involved in a consumption trouble will increase more and more.

6.We should press on with supplying care and/or welfare facilities newly.

7.More people to carry nursing care and welfare are needed.

8.Society nursing care facilities with autonomous communities as the leader should be productive.

9. From now on, it is possible to think that only the elderly needing serious care and a single elderly enable to be cared.

10. Education is needed for elderly to live independently as one consumer.

11. The people should pay more fees related to the social security.

12. We should fundamentally reconsider the previous policy of the care and welfare.

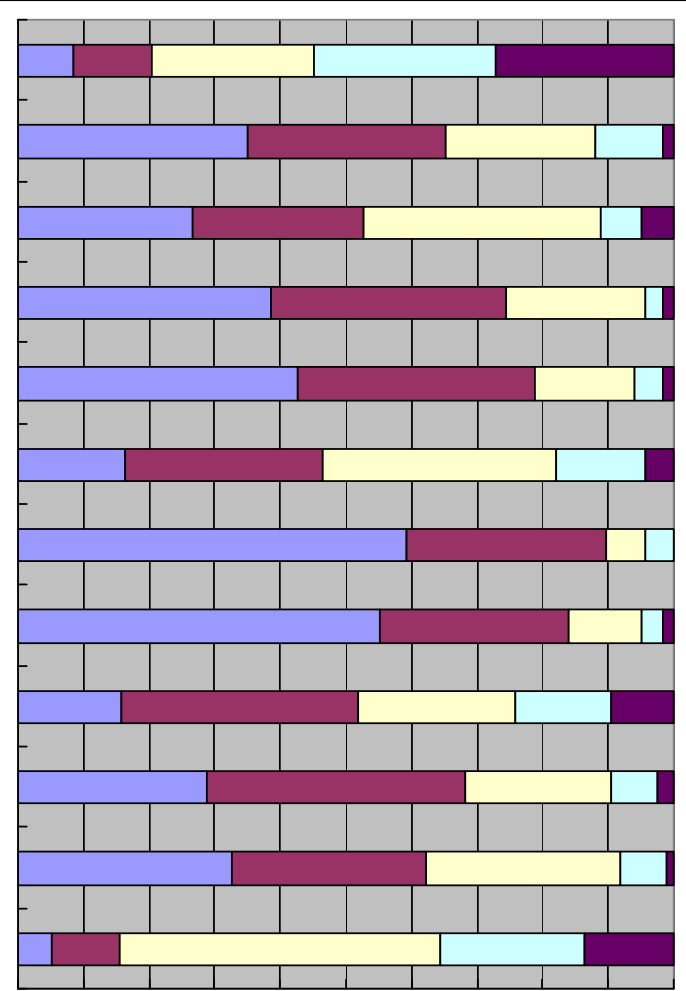

$\begin{array}{lllllllllll}0 \% & 10 \% & 20 \% & 30 \% & 40 \% & 50 \% & 60 \% & 70 \% & 80 \% & 90 \% & 100 \%\end{array}$

$\square$ agree $\square$ agree a little $\square$ yes and no $\square$ disagree not too $\square$ disagree

Fig.1 Perspective and present conditions of nursing care and welfares

1. Catching their consumer problem from the user and organizing the fact as a data

2. Catching their consumer problem from the user, but not organizing the fact as a data

3. Catching users' buying activities from their family and organizing the fact as a data

4. Catching users' buying activities from their family, but not organizing the fact as a data

5. Not catching users' buying activities, but being consulted about their subjects

6. Office staffs and users sometimes talking about their buying activities

7. Users sometimes talking about their consumer problem

8. Not mentioning about their consumption problem or buying activities in the facilities

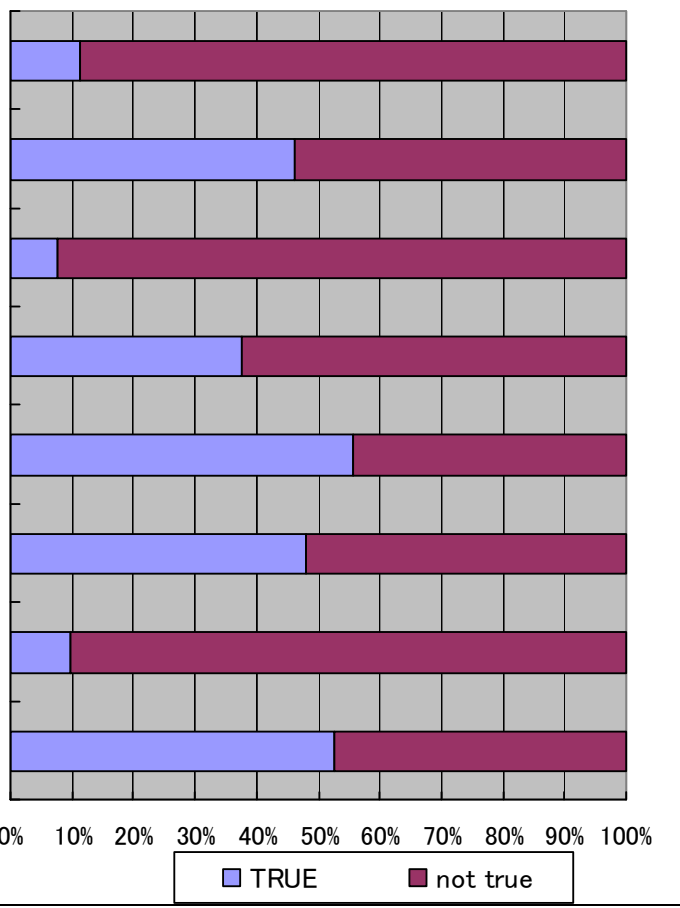

Fig.2 Present condition of keeping track of consumer problems or shopping activities of elderly 


\subsection{Actual condition of consumer problems}

At present, in Japan, consumption crimes targeting the elderly or females are occurring frequently, and are a big social problem. The figure 3 shows what was examined concerning the degree of 12 terms. Referring to the shopping activities or consumption problems of the elderly, the social welfare facilities keeping track on "2. Have bought something you don't need" and "12. Forgetting that you have already bought it, and bought the same one" are about 25 to $30 \%$, but in present, we may know that there aren't so many in general.

But, as seen in the first question (referring to the state of society) from now on, it is expected that the cases of elderly being involved in consumption troubles will increase. Furthermore, it is needed to observe closely the fact that the socioeconomic factor such as business conditions and social crimes are related.

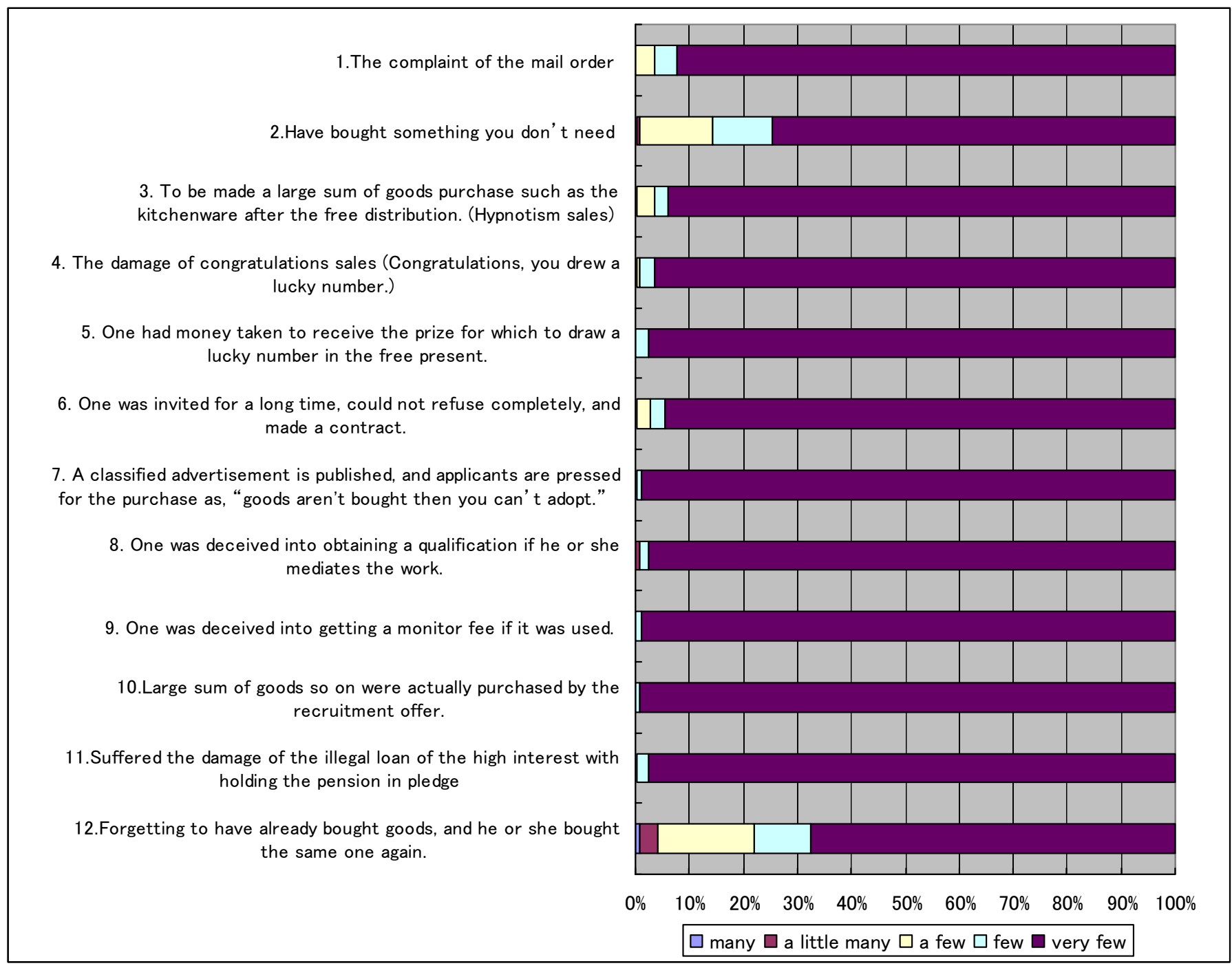

Fig.3 The actual condition of consumer problems

\subsection{Need of perspective consumer education}

The figure 4 is a survey result about the need of perspective consumption education. Although present execution conditions according to the same term is also researched, the truth is that in any of the terms they are hardly being carried out. As we can see about $40 \%$ in either term that it is hard to determine now. 
"Want to carry it out" or "Will examine" are generally few, and except for "2.Personal experience" that was $20 \%$, are mostly about 10 to $15 \%$. Also, regarding to "7. Game using computers", it is only $5 \%$ with "want to carry it out" and "will examine" together.

From the fact that the "Consumption educations are needed" was about $70 \%$ in the first question, it can be focused on the point that none of the ideas has become specific about concrete education methods or means.

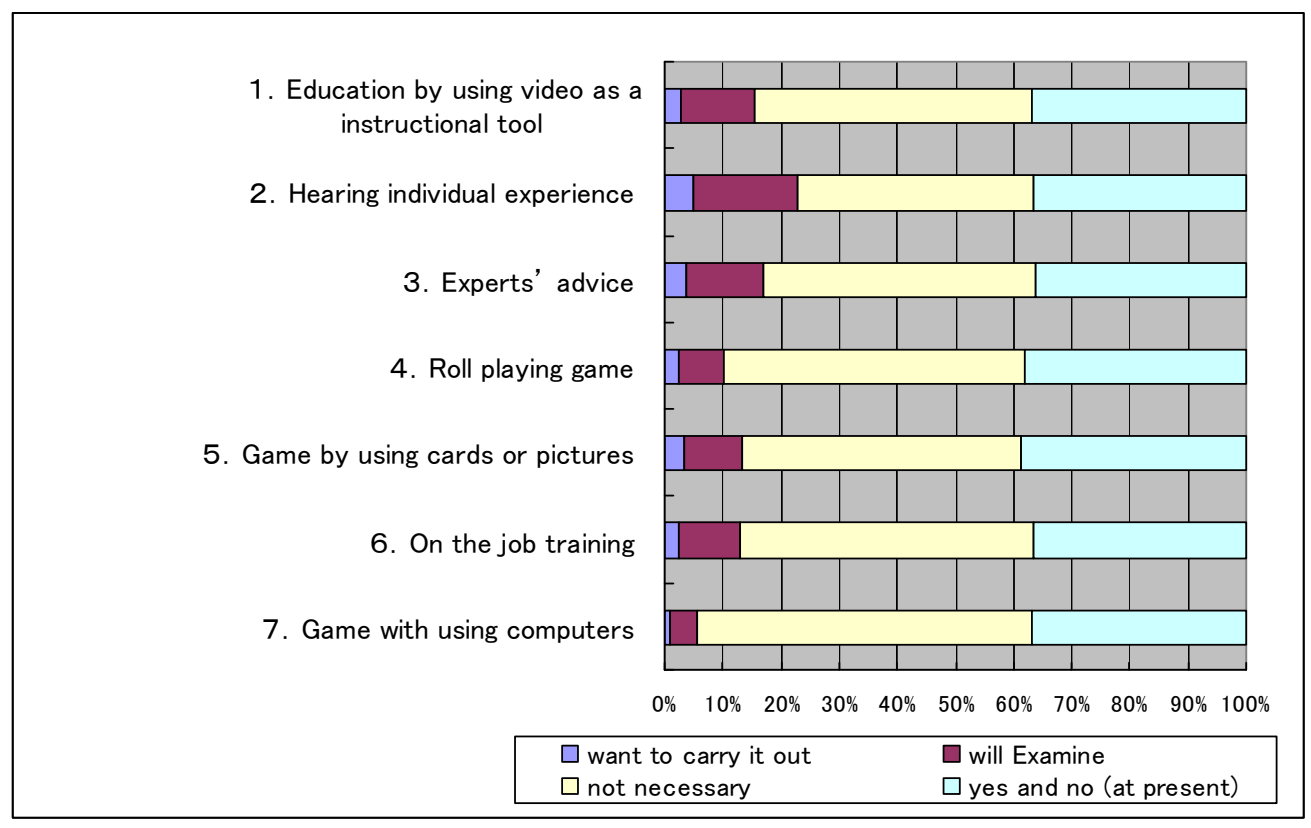

Fig.4 Need of perspective consumer education

\subsection{Correspondence of dementia}

By this investigation, related questions were done to the slight dementia too, but the figure 5 is a part of it. By the way, the main measures for determination of dementia are Hasegawa method and of nursing care insurance.

The execution rate of the "mental training" is $17 \%$. And the present condition, the actual work on the consumption problems and its countermeasure or education is rarely done comparing with the cases of general elderly.
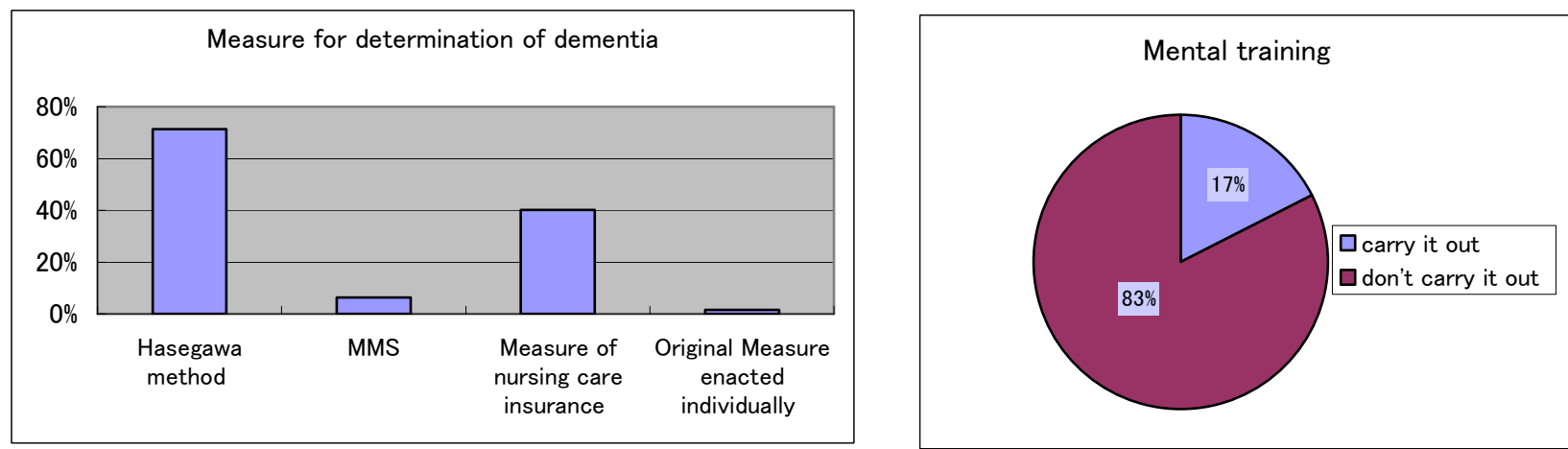

Fig.5 Correspondence to dementia

\subsection{About Takizawa program and motivative exercise}

We investigated about both the degree of recognition and the degree of concern of the Takizawa program and 
motivative exercise as a related research.

The figure 6 shown below is a result about the degree of recognition. Until now, we verified the validity of the Takizawa program and motivative exercise. We can confirm that the degree of recognition is very low through this result of investigation. The degree of recognition for anyone of "the validity", "the effect of the walker with the sled" and "physical therapist of 1 to many patients" doesn't meet 10\%, either.

On the other hand, as for the degree of the concern (fig.7), when we put "It is a little interested." together "It is interested." then it is about $50 \%$. The person in charge of the rehabilitation spot did not satisfy with the present condition but expected the method of the new rehabilitation as to another our research.

From this, we want to continue the activities for the diffusion of the Takizawa program and motivative exercise.

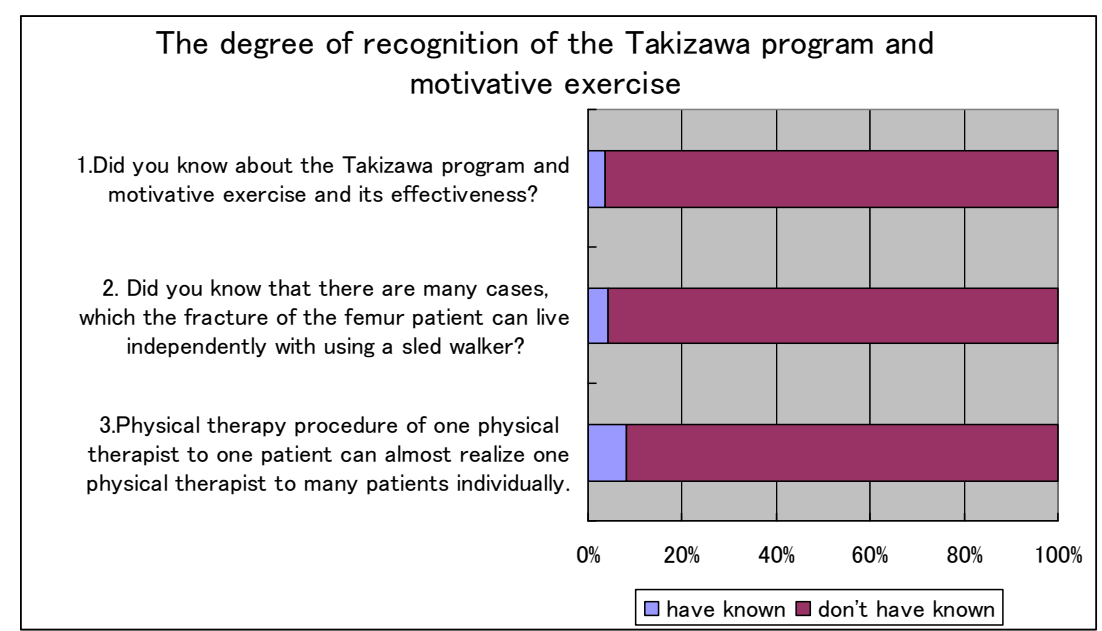

Fig.6 Degree of recognition of the Takizawa program and motivative exercise

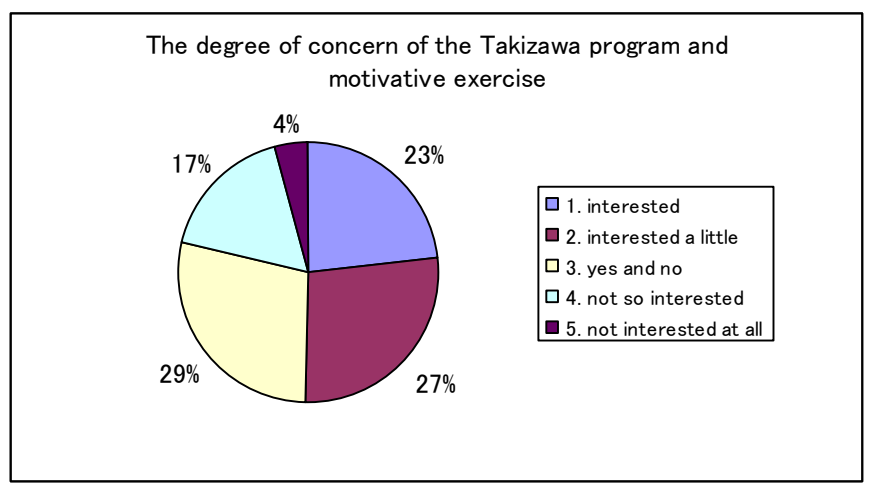

Fig.7 Degree of concern of the Takizawa program and motivative exercise

\subsection{Results of the mail survey}

Putting together the results of the questionnaire survey, we can state as follows:

1:Considering the future state of society, with the crunch of social security-related expenditure as its reason, social welfare facilities will be short.

2:Therefore, a consumption education for the elderly to live independently will be needed.

3:Also, we should enrich the nursing care of the society (neighborhood association), and increase the number of 
people talented for welfare and nursing care.

4:However, at present, at the nursing care and welfare facilities, it can't be said that education for elderly be done, and the truth is that concrete ideas in perspective are in short.

5:People of the facility thought it as a future examination subject. (It can be said that it is harder for the elderly with dementia.)

\section{A future plan}

We expect continuing fosterage from Ministry of technology and education and other related organizations for a continuation research. And in such condition, we are thinking of continuing the research in the following aspects:

The present condition of consumption education for elderly in Japan is as mentioned above, but we would like to compare and research this result with the present condition of the United States of America and other countries. Also, we would like to investigate on advanced education practices, too.

Moreover, it is also a subject to develop consumption education methods or programs as a practical matter based on the result of the trial in this research about the consumption education proceeded.

Furthermore, we wish to propose a better method in verifying the result. To reach the goal, our plan is to promote the activities of more academic conferences locally, nationally and internationally in order to gain understanding, knowledge, cooperation and collaboration with our own system and with other countries.

\section{References}

1) Kenji Ushizawa, The research of the reality and the future of the consumer education to the elderly, Proc. of the 2 nd International Biophilia Rehabilitation Conference, Canada, 2003.

2) Hiroshi Nagasawa, Kenji Ushizawa, Shigeo Takizawa, et al., Consumption behavior and its influence on their physical and mental function of fragile elderly and elderly with slight dementia, Proc. of the 2nd International Biophilia Rehabilitation Conference, Canada, 2003.

3) Shigeo Takizawa, Nobuo Aoki, Rika Wada, Kenji Ushizawa, et al., Approach and Trial of the New ADL Evaluation Chart for Elderly Patients with Consumption Behavior Viewpoint Adopted, Proc. of the 2nd International Biophilia Rehabilitation Conference, Canada, 2003.

4) Shigeo Takizawa and Kenji Ushizawa, Consumer behavior in aged society (in Japanese), Proc. of the 7th Biophilia Rehabilitation Conference, Oita, 2003.

5) Takizawa, Shigeo, Tetsuhiko Kimura, Hideo Kijima, Yuzou Okamoto, Kentaro Nagaoka, Kyoko Takizawa, The development of devices for the MOTIVATIVE exercise of impaired extremities, CSUN's sixteenth Annual International Conference, Los Angeles, 2003.5.24, 2000.

http://www.csun.edu/cod/conf/2000/proceedings/csun00.htm 
